\title{
SITUAÇÃO EPIDEMIOLÓGICA DA TRANSMISSÃO DA COVID-19 NO NORTE DE MINAS GERAIS, BRASIL
}

\section{EPIDEMIOLOGICAL SITUATION OF COVID-19 TRANSMISSION IN NORTHERN MINAS GERAIS, BRAZIL}

\section{Sandra Célia Muniz Magalhães} Doutora em Geografia Universidade Federal de Uberlândia sandramunizgeo@hotmail.com

Flávia de Oliveira Santos Doutora em Geografia, Instituto de Geografia Universidade Federal de Uberlândia flavia.santos@ufu.br

Samuel do Carmo Lima Doutor em Geografia, Instituto de Geografia Universidade Federal de Uberlândia samuel@ufu.br

Elivelton da Silva Fonseca Doutor em Geografia, Instituto de Geografia Universidade Federal de Uberlândia elivelton.fonseca@gmail.com

\section{RESUMO}

O Brasil é um país com extensa dimensão territorial e com acentuadas desigualdades socioeconômicas entre suas regiões, o que reflete diretamente no acesso à saúde, principalmente da população mais carente. O mesmo ocorre nos estados, como é o caso de Minas Gerais, com diversidade regional, com características sociais, econômicas e culturais variadas. Daí a importância de se promoverem estudos de cunho regional na tentativa de evidenciar as potencialidades e fragilidades que as regiões apresentam, para que, assim, as políticas públicas de saúde possam ser desenvolvidas com maior eficácia, possibilitando as autoridades sanitárias ações mais assertivas no combate a COVID-19. Em face a essa realidade, o presente artigo tem como objetivo apresentar uma reflexão sobre a situação epidemiológica da transmissão da covid-19 no norte de Minas Gerais, Brasil. Considerando que a pandemia de Covid-19 ainda não chegou definitivamente ao Norte de Minas, e que a relação leitos de UTI/população não é adequada, isso impõe ao Estado e às autoridades sanitárias da Região de Saúde tomar medidas urgentes para ampliar o número de leitos com respiradores para atender a demanda crescente que se espera, quando a região enfrentar o pico da pandemia. Outra questão importante é que a hora de estabelecer medidas que imponham o isolamento social com mais rigor é agora, para achatar a curva de transmissão, antes que o número de casos da doença cresça e a situação fique fora de controle.
\end{abstract}

Palavras-chave: COVID-19. Unidades Regionais de saúde. Leitos de UTI. Norte de Minas Gerais.

\section{ABSTRACT}

Brazil is a country with an extensive territorial dimension and with a lot of socioeconomic inequalities between its regions, which directly reflects in access to health, mostly of the

Recebido em: 28/04/2020

Aceito para publicação em: 02/06/2020. 
poorest population. The same occurs in the states, as is the case in Minas Gerais, with regional diversity, with varied social, economic and cultural characteristics. So the importance of promoting studies of a regional nature in an attempt to highlight the strengths and weaknesses that the regions present, so that public health policies can be developed more effectively, enabling health authorities to take more assertive actions in the fight against COVID -19. In view of this reality, this article aims to present a reflection on the epidemiological situation of the transmission of covid-19 in northern Minas Gerais, Brazil. Considering that the Covid-19 pandemic has not yet definitively reached the North of Minas Gerais, and that the ICU beds / population ratio is not adequate, this requires the State and the health authorities of the Health Region to take urgent measures to increase the number of beds with respirators to meet the growing demand expected when the region faces the peak of the pandemic. Another important issue is that the time to establish measures to impose social isolation more rigorously is now, to flatten the transmission curve, before the number of cases of the disease grows and the situation gets out of control.

Keywords: COVID-19. Regional health units. ICU beds. North of Minas Gerais.

\section{INTRODUÇÃO}

Os primeiros coronavírus humanos foram isolados pela primeira vez em 1937. No entanto, foi em 1965 que o vírus foi descrito, em decorrência do perfil na microscopia, parecendo uma coroa. O novo agente do coronavírus surgiu em Wuhan, na China, em 31 de dezembro de 2019, com uma capacidade de infecção e propagação impressionante. Em 30 de janeiro, a Organização Mundial de Saúde (OMS) declarou a epidemia uma emergência internacional (PHEIC) 4. Ao final do mês de janeiro, diversos países já haviam confirmado importações de caso, incluindo Estados Unidos, Canadá e Austrália. No Brasil, em 7 de fevereiro, havia 9 casos em investigação, mas sem registros de casos confirmados (BRASIL, 2020).

A primeira ocorrência foi registrada no Brasil em 26/02/2020, sendo confirmada a primeira morte em $17 / 03 / 2020$, quando já havia 921 casos confirmados. O paciente que veio à óbito era um homem da cidade de São Paulo, de 62 anos.

Até 09/05/2020, já havia no país 155.939 casos confirmados e 10.627 óbitos por Covid-19 confirmados. Em Minas Gerais, até 09/05/2020 havia 3.123 casos confirmados (casos + óbitos), com 118 óbitos de Covid-19, tendo ainda 747 óbitos suspeitos, em investigação (BRASIL, 2020, MINAS GERAIS, 2020).

Em 11 de março, a OMS elevou o estágio de preocupação e declarou pandemia do novo coronovírus (SARS-CoV-2) que provoca a doença chamada Covid-19. O termo "pandemia" significa que a circulação está ocorrendo em todo o mundo simultaneamente e, não está mais localizada em uma única região do globo (RADIS, 2020).

Diferentemente das epidemias que surgiram na China em 2002 e no México em 2009, o novo coronavírus combina rápida expansão e um acesso fácil a diferentes pontos do globo, o que se explica pela posição da China na atual economia mundial e pelas redes de conexões aéreas que terminaram por impulsionar a difusão do vírus. De uma espacialização que se confunde com as principais rotas aéreas, o novo coronavírus agora aterrissa no cotidiano das cidades e mira as periferias (CASTILHO, 2020).

O vírus da geopolítica, aquele que viajou de avião, agora alcança os lugares e invade o cotidiano dos pobres. Assim como a globalização, o coronavírus também chega às periferias. Nesse ponto, a metáfora se repete, pois em seu DNA germina a perversidade crua, impiedosa, insana. (CASTILHO, 2020, p. 3).

O Brasil é um país continental, com acentuadas desigualdades socioeconômicas, com populações vulneráveis em todas as regiões, e desigualdades entre as regiões. É o que ocorre no estado de Minas Gerais, um vasto território, com diferenças regionais que se estabelecem por uma variação de condições sociais, econômicas e culturais, que afetam diretamente o acesso aos serviços de saúde das populações mais pobres.

A potencial chegada do novo vírus coloca à prova a estrutura de vigilância existente no país, principalmente num momento em que a redução de investimentos no Sistema Único de Saúde (SUS) 
e na pesquisa fragiliza a capacidade de detecção precoce e de resposta. O Brasil, que foi protagonista na epidemia de Zika, precisa acompanhar o avanço de conhecimentos gerados no exterior e preparar-se para as pesquisas e demandas específicas que surgirão no país, incluindo diagnóstico, assistência, prevenção e promoção da saúde. (LANA et al, 2020, p. 3).

A Covid-19 é uma doença que apresenta um quadro clínico que varia de infecções assintomáticas a quadros respiratórios graves. De acordo com a OMS, a maioria dos pacientes com Covid-19 (cerca de $80 \%$ ) podem ser assintomáticos e cerca de $20 \%$ dos casos podem requerer atendimento hospitalar por apresentarem dificuldade respiratória e desses casos aproximadamente $5 \%$ podem necessitar de suporte para o tratamento de insuficiência respiratória, suporte ventilatório (BRASIL, 2020).

Ferguson et al. (2020) destacam que a identificação, o controle e isolamento dos casos suspeitos, a quarentena domiciliar e o distanciamento social dos idosos e indivíduos de grupos de risco podem reduzir o pico da demanda de assistência médica em até dois terços e o número de mortes pela metade. Nesse sentido, o objetivo deste estudo apresentar uma reflexão sobre a situação epidemiológica da transmissão da covid-19 no norte de Minas Gerais, Brasil.

\section{O TERRITÓRIO DA SAÚDE NO NORTE DE MINAS}

O Norte de Minas possui uma população de 1.684.710 habitantes, com uma densidade demográfica de 13,10 hab./ $\mathrm{Km}^{2}$. Há uma população rural relativamente alta comparada as demais regiões do Estado de Minas Gerais (IBGE, 2019). Outra característica que chama a atenção é a grande desigualdade social. A região apresenta, ainda, baixos indicadores socioeconômicos e a maioria dos municípios é dotada de deficiente infraestrutura de serviços sociais básicos, fatores determinantes para a formação do quadro de elevada pobreza e de exclusão social.

Um dos grandes problemas da região diz respeito às doenças relacionadas à ineficiência ou inexistência do saneamento básico, com alta prevalência de esquistossomose em alguns municípios. Também, há alta prevalência de doenças de chaga, principalmente na população mais velha. O que fica em realce e chama a atenção é a reduzida oferta de serviços de saúde que não supre as demandas da população (MAGALHAES; LIMA, 2012).

Montes Claros é a cidade que possui a melhor infraestrutura de saúde do Norte de Minas. Possui quatro hospitais de grande porte, um de médio e um de pequeno porte que atende a demanda de urgência e emergência de Montes Claros, procedimentos referenciados de outros municípios, além do acolhimento a pacientes que vêm de demanda espontânea. O município possui equipamentos de alta resolução para a realização de quase todos os processos preconizados pelo SUS, inclusive transplantes, o que justifica a posição de polo regional, que se comprova pela forte polarização que a cidade exerce sobre a região, para onde aflui o grande fluxo de pessoas em busca de serviços diversos, incluindo-se os serviços de saúde de maior complexidade (Figura 1).

Analisando ainda a espacialização dos serviços de saúde no Norte de Minas, é possível perceber que a maioria, ou seja, 53 dos 86 municípios da região possuem apenas os procedimentos ambulatoriais (atenção básica), sendo necessário recorrer a outros polos quando necessitam de serviços hospitalares (atenção secundária e terciária).

Os municípios das microrregiões de Bocaiúva, Brasília de Minas, Grão Mogol, Janaúba, Pirapora, Salinas, São Francisco e Taiobeiras possuem hospitais de média complexidade, que atendem aos municípios de suas áreas de abrangência.

Os municípios de Buritizeiro, Capitão Enéias, Coração de Jesus, Espinosa, Francisco Sá, Jaíba, Januária, Joaquim Felício, Juramento, Manga, Monte Azul, Porteirinha, Rio Pardo de Minas, São João do Paraíso, São Romão, Urucuia e Varzelândia têm o hospital de pequeno porte que oferece os serviços ambulatoriais e hospitalares de baixa complexidade. Os demais municípios possuem apenas as Unidades Básicas de Saúde que realizam os procedimentos de atenção primária.

Até o dia 06/05/2020, 50 casos de Covid-19 foram confirmados na região no Norte de Minas, distribuídos da seguinte forma: Montes Claros 21, São Francisco 10, Brasília de Minas 5, Janaúba 3, Pirapora 2, São Romão 2, e Espinosa, Ibiaí, Icaraí de Minas, Itacarambi, Monte Azul, Porteirinha e Varzelândia, 1 caso confirmado em cada um desses municípios (Figura 2). Entretanto, são muitos os casos em investigação, somente em Montes Claros são 407 casos a espera de resultados (MINAS GERAIS, 2020). 
Figura 1 - Polarização de Montes Claros nos serviços de saúde da região Norte de Minas

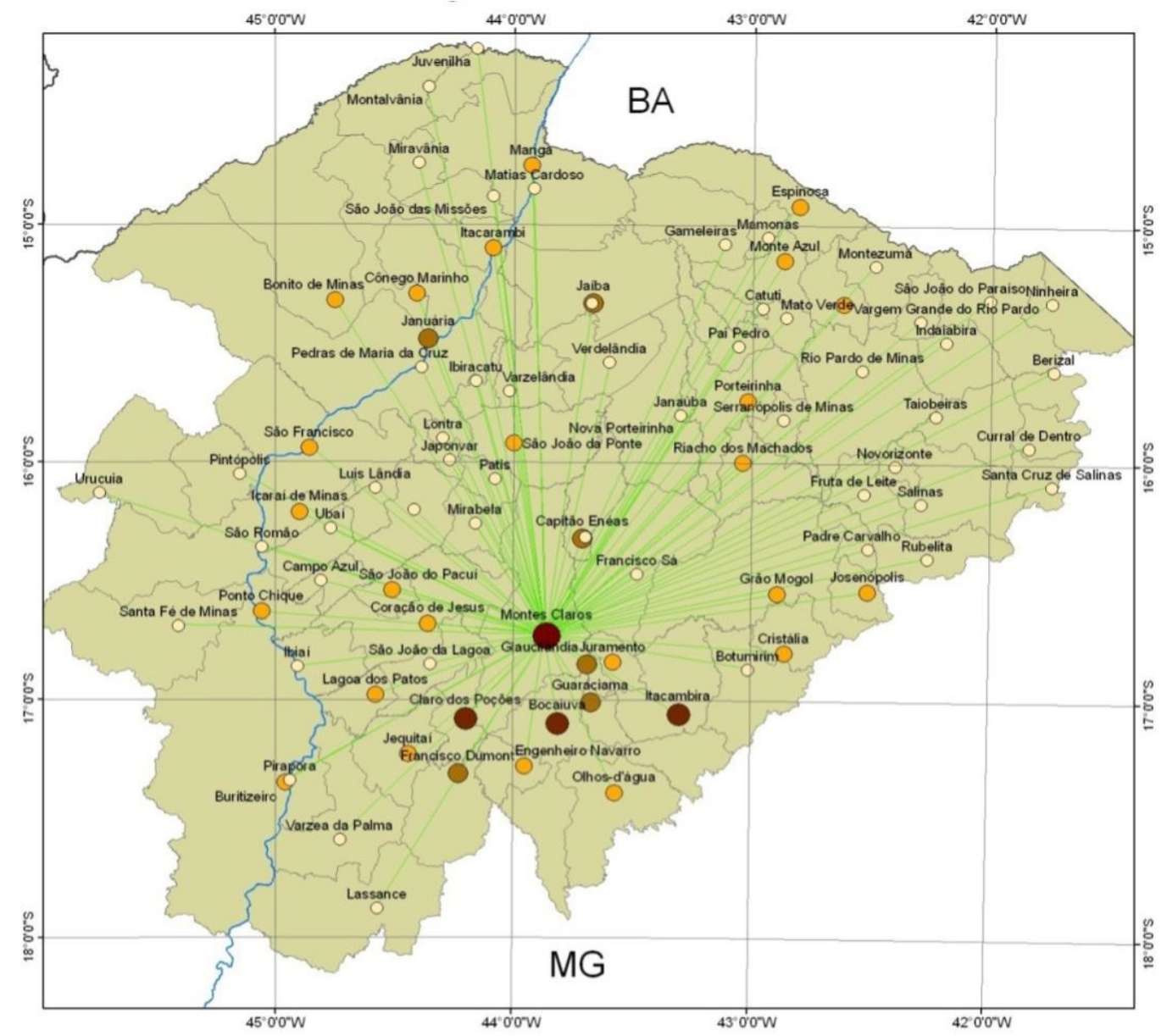

$\mathrm{N}^{\circ}$ de atendimentos

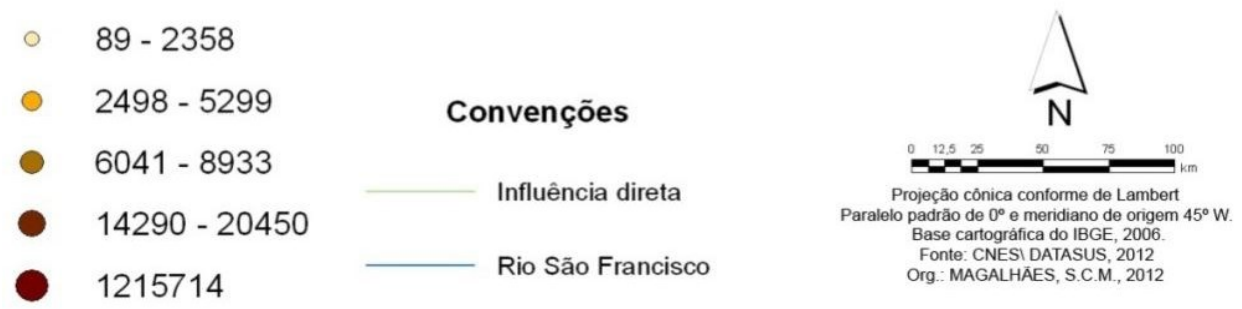

Conforme aponta o Quadro 1, até o dia 07/05/2020, foram confirmados 7 (sete) óbitos por Coivd-19 na região, sendo 2 em Montes Claros, 2 em São Francisco, 1 em São Romão, 1 em Espinosa e 1 em Varzelândia. Os pacientes que vieram a óbito, na maioria eram pacientes do sexo masculino, todos com idade superior a 50 anos e apenas 1 não possuía comorbidades (MINAS GERAIS, 2020).

Para reforçar as ações de enfrentamento ao novo coronavírus, os 86 municípios que integram a região de saúde do Norte de Minas receberam do Ministério da Saúde mais de $R \$ 32,3$ milhões. Entre os municípios da macrorregião que receberam o maior aporte financeiro estão: Montes Claros com R\$ 15,5 milhões; Janaúba (R\$ 2,106 milhões); Pirapora (R\$ 1,408 milhão); Brasília de Minas (R\$ 1,146 milhão) e Taiobeiras (R\$ 1,221 milhão) (MINAS GERAIS, 2020). 
Quadro 1 - Óbitos COVID-19 na macrorregião de saúde do norte de Minas Gerais, em 09/05/2020

\begin{tabular}{llllll}
\hline Paciente & Sexo & Idade & Município de residência & Data do óbito & Comorbidade \\
\hline 1 & $\mathrm{M}$ & 69 & Montes Claros & $01 / 04 / 2020$ & Não \\
2 & $\mathrm{M}$ & 55 & Espinosa & $12 / 04 / 2020$ & $\mathrm{Sim}$ \\
3 & $\mathrm{~F}$ & 75 & Varzelândia & $16 / 04 / 2020$ & $\mathrm{Sim}$ \\
4 & $\mathrm{M}$ & 75 & São Francisco & $17 / 04 / 2020$ & $\mathrm{Sim}$ \\
5 & $\mathrm{M}$ & 74 & São Romão & $24 / 04 / 2020$ & Não \\
6 & $\mathrm{M}$ & 75 & São Francisco & $22 / 04 / 2020$ & $\mathrm{Sim}$ \\
7 & $\mathrm{M}$ & 91 & Montes Claros & $29 / 04 / 2020$ & $\mathrm{Sim}$ \\
\hline \multicolumn{7}{r}{ Fonte: CIEVS-Minas/COES MINAS/COVID-19/SES-MG } & Org.: MAGALHÃES, 2020.
\end{tabular}

Outros municípios também receberam recursos consideráveis para o enfrentamento do novo coronavírus, com destaque para: Salinas ( $R \$ 612$ mil); Jaíba (535 mil); Januária ( $R \$ 486$ mil); Varzelândia (R\$ 398 mil); Francisco Sá (R\$ 349 mil); Porteirinha ( $R 314$ mil); Bocaiúva (314 mil); Coração de Jesus (R 267 mil); Monte Azul (R\$ 261 mil); Montalvânia (R 244 mil); Várzea da Palma (R\$ 216 mil); Grão Mogol (R\$ 213 mil) e São João das Missões (210 mil). De acordo com O Ministério da Saúde (2020), essa verba será fundamental para a "[...] aquisição de materiais e insumos, abertura de leitos, além do custeio de profissionais de saúde, ações e procedimentos, de acordo com a necessidade local, para enfrentamento específico ao coronavírus" (MINAS GERAIS, 2020)

Figura 2 - Casos confirmados de COVID-19 na Região de saúde no norte de Minas Gerais, 6 de maio de 2020

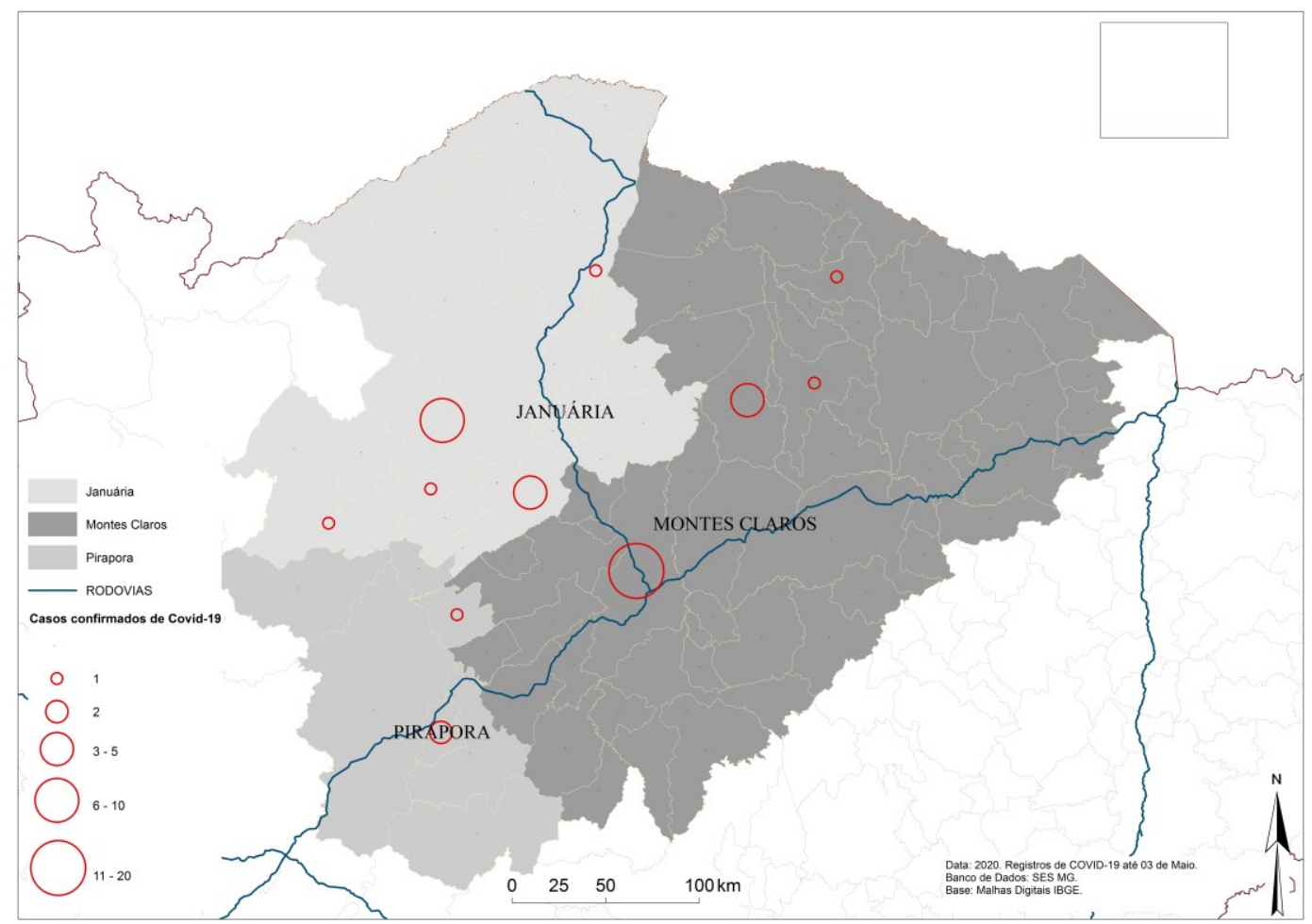

Fonte: Secretaria de Estado de Saúde de Minas Gerais, 2020.

Diante do quadro epidemiológico que tende a se agravar com o avanço da pandemia na região, avaliou-se a infraestrutura de leitos de UTI na Superintendência Regional de Montes Claros, e nas Gerências Regionais de Saúde de Januária e Pirapora, que abrangem 86 municípios, distribuídos em 
nove microrregiões de Saúde. A SRS de Montes Claros é composta por cinco microrregiões, 54 unidades de saúde. A GRS de Januária, com 3 microrregiões, conta com 25 unidades de saúde e a GRS Pirapora, com apenas 1 micro região, conta com 7 unidades de saúde (Tabela 1).

Com relação à quantidade de leitos de UTI, observa-se que na área de abrangência da SRS de Montes Claros há uma maior quantidade de leitos de UTI (145), entretanto, uma maior população (1.130.030), apresenta uma relação de $1,3 \mathrm{UTI} / 10.000 \mathrm{hab}$, considerada adequada no limite da recomendação da OMS, de 1 a 3 leitos/10 mil hab. (RADIS, 2020). Mas, a condição se agrava quando se considera o fluxo de polarização de Montes Claros em todo o Norte de Minas, para além da área de abrangência da própria SRS (Figura 1).

Tabela 1 - Unidades Regionais de Saúde no Norte de Minas Gerais

\begin{tabular}{|c|c|c|c|c|c|}
\hline Municípios & Microrregião & $\begin{array}{l}\text { Unidades } \\
\text { Regionais de } \\
\text { Saúde }\end{array}$ & População & UTI & $\begin{array}{c}\text { UTI /POP } \\
(10.000)\end{array}$ \\
\hline Montes Claros & 5 & 54 & 1.130 .030 & 145 & 1,3 \\
\hline Januária & 3 & 25 & 407.689 & 30 & 0,7 \\
\hline Pirapora & 1 & 7 & 146.991 & 20 & 1,4 \\
\hline TOTAL & 9 & 86 & 1.684 .710 & 195 & 1,2 \\
\hline
\end{tabular}

$\mathrm{Na}$ área de abrangência da GRS de Pirapora, que possui menor quantidade de leitos de UTI (20), porém cobre um total menor de habitantes (146.991), tem relação UTI/POP (10.000) semelhante a da GRS de Montes Claros, ou seja a situação é tão grave quanto. Na área de abrangência da GRS de Januária, a relação UTI/POP (10.000) é de 0,7 situação mais agravada que na área da SRS de Montes Claros, o que significa que, em caso de agravamento da pandemia, as previsões são mais sombrias ainda, tendo em vista que em $5 \%$ dos casos, os pacientes podem necessitar de leitos de UTI com suporte ventilatório para o tratamento de insuficiência respiratória.

Rache et al (2020), dizem que o Sistema Único de Saúde (SUS), em $72 \%$ das regiões de saúde do país (279 de 436), não possuem o número de leitos de UTI necessários, nem mesmo para um ano típico, situação que está muitíssimo agravada com a pandemia, que afeta a maioria dos países do mundo.

Todos os países estão indo ao mercado internacional (China) para comprar insumos e equipamentos, principalmente, respiradores e não há oferta que atenda a toda essa demanda. Isso nos coloca em condições de esperar pelo pior, porque as medidas de isolamento social, que podem reduzir a contaminação e achatar a curva de transmissão não estão sendo tomadas.

Os municípios que receberam recursos financeiros do governo federal para o enfrentamento da pandemia devem usá-los com transparência, divulgando à população o destino de sua aplicação, que devem ser definidos pela secretaria de saúde, assessorada por um comitê de profissionais de saúde pública.

As previsões para as próximas semanas são de que a situação epidemiológica da Covi-19 deve se agravar nas pequenas cidades do interior do Brasil. Neste municípios, o sistema de saúde deve entrar em colapso. Deste modo, as intervenções das autoridades sanitárias para enfrentarem o agravamento da pandemia devem combinar estratégias de desaceleração da expansão da transmissão do vírus, com o reforço da infraestrutura hospitalar com ampliação de leitos de UTI, equipamentos de proteção individual para os profissionais de saúde e, ainda, na contratação de profissionais de saúde, quando for necessário.

\section{CONCLUSÃO}

Entende-se que a difusão da Covid-19 no Brasil, que começou pelas grandes metrópoles que têm alta conectividade com o exterior, pelos bairros da classe média e de alta renda, para em seguida atingir os bairros da periferia, de populações pobres e vulneráveis. A maioria das capitais do país já apresentam sistemas de saúde colapsados, com pacientes morrendo em casa, sem assistência. A próxima etapa da difusão da pandemia já começou atingindo as cidades pequenas. 
Certamente, as consequências da pandemia são diferenciadas entre as regiões do país. Em cada lugar, dependendo das características socioeconômicas, do nível de vulnerabilidade social da população, mas espera-se que os municípios mais afetados sejam aqueles que negligenciam as medidas de isolamento social, em detrimento da saúde, dando prioridade à economia.

Nos municípios pequenos da região, que ainda não estão sendo muito afetados pela pandemia, caso não tomem as medidas necessárias de proteção da saúde e da vida, verão nos próximos dias ou semanas uma acelerada ampliação da demanda por serviços de saúde, principalmente de leitos hospitalares de UTI, com suporte ventilatório mecânico para pacientes com quadros de síndrome respiratória aguda provocados pela Covid-19, que serão buscados nas unidades regionais de saúde de Pirapora, Januária e Montes Claros, que apresentam déficit de serviços de saúde.

As autoridades sanitárias do Estado e dos municípios da região precisam tomar as medidas necessárias imediatamente para reduzir a taxa de transmissão e ainda, avaliando a infraestrutura de saúde, ampliar a quantidade de leitos de UTI, assim como contratar recursos humanos qualificados (médicos, enfermeiros, técnicos de enfermagem, fisioterapeutas, etc.)

\section{REFERÊNCIAS}

BRASIL. Painel de casos de doença pelo coronavírus 2019 (COVID-19). Ministério da Saúde. Brasília: 2020. Disponível em https://covid.saude.gov.br/. Acesso em 07 de maio de 2020.

BRASILInfecção humana pelo novo coronavírus (2019-nCoV). Boletim Epidemiológico 2020, 02 . . Secretaria de Vigilância em Saúde, Ministério da Saúde. Brasília: 2020. Disponível em: https://portalarquivos2.saude.gov. br/images/pdf/2020/fevereiro/07/BE-COECoronavirus-n020702.pdf. Acesso em 07 de maio de 2020.

CASTILHO, Denis. Um vírus com DNA da globalização: o espectro da perversidade. Pragmatismo político. 23 de março de 2020. Disponível em: https://www.pragmatismopolitico.com.br/2020/03/virusdna-globalizacao-perversidade.html. Acesso em 04 de maio de 2020. ttps://doi.org/10.4000/espacoeconomia.10332

IBGE. Censo populacional 2010. Estimativa, 2019. Instituto Brasileiro de Geografia e Estatística. Rio de Janeiro: 2010. Disponível em: <http://www.ibge.gov.br/home/>. Acesso em: 21 de março de 2016.

FERGUSON, Neil M. et al. Impact of non-pharmaceutical interventions (NPIs) to reduce COVID-19 mortality and healthcare demand. Imperial College COVID-19 Response Team. 16 March 2020. Disponível em https://dsprdpub.cc.ic.ac.uk:8443/handle/10044/1/77482. Acesso em: 04 maio 2020.

LANA, Raquel Martins et al. Emergência do novo coronavírus (SARS-CoV-2) e o papel de uma vigilância nacional em saúde oportuna e efetiva. Cadernos de Saúde Pública, 2020; v. 36, n. 3, Rio de Janeiro, março de 2020. Disponível em: http://cadernos.ensp.fiocruz.br/csp/artigo/999/emergnciado-novo-coronavrus-sars-cov-2-e-o-papel-de-uma-vigilncia-nacional-em-sade-oportuna-e-efetiva. Acesso em 04 de maio de 2020. https://doi.org/10.1590/0102-311x00019620

MAGALHÃES, Sandra Célia Muniz. Fatores determinantes na ocorrência de tuberculose no Norte de Minas Gerais / Sandra Célia Muniz Magalhães, Samuel do Carmo Lima - Montes Claros: Unimontes, 2013.

PEREIRA, Anete Marília; SOARES, Beatriz Ribeiro. Montes Claros e sua região: novas espacialidades, velhos problemas. X Encontro de Geógrafos da América Latina, 20 a 26 de março de 2005. Anais. Universidade de São Paulo. Disponível em: http://observatoriogeograficoamericalatina.org.mx/egal10/Geografiasocioeconomica/Geografiaregional 123.pdf. Acesso em: 02 de maio de 2020.

RACHE, Beatriz et al. Necessidades de Infraestrutura do SUS em Preparo ao COVID-19: Leitos de UTI, Respiradores e Ocupação Hospitalar. Nota Técnica n.3. IEPS: São Paulo, 2020.

MINAS GERAIS. Boletim Epidemiológico. Secretaria de Estado da Saúde de Minas Gerais. Disponível em: www.saude.mg.gov.br. Acesso em 30/04/2020.

MINAS GERAIS. Municípios da região ampliada de saúde do Norte de Minas recebem R $\$ 32,3$ milhões para enfrentamento ao coronavírus. Notícias, 10/04/2020. Secretaria de Estado de Saúde de Minas Gerais, 2020b. Disponível em: <https://www.saude.mg.gov.br/component/gmg/story/12457- 
municipios-da-regiao-ampliada-de-saude-do-norte-de-minas-recebem-r-32-3-milhoes-paraenfrentamento-ao-coronavirus>.

WHO. IHR procedures concerning public health emergencies of international concern (PHEIC). World Health Organization.Disponível em: http://www.who. int/ihr/procedures/pheic/en/. Acesso em 29/01/2020. 